\title{
Interaction of an along-shore propagating vortex with a vortex enclosed in a circular bay
}

\author{
Eugene A. Ryzhov, ${ }^{1, a)}$ Konstantin V. Koshel, ${ }^{1, b)}$ Mikhail A. Sokolovskiy, ${ }^{2,3, c)}$ \\ and Xavier Carton ${ }^{4, d)}$ \\ ${ }^{1}$ V.I.Il'ichev Pacific Oceanological Institute of FEB RAS, 43, Baltiyskaya Street, Vladivostok 690041, Russia \\ ${ }^{2}$ Water Problems Institute of RAS, 3, Gubkina Street, Moscow 119333, Russia \\ ${ }^{3}$ P.P. Shirshov Institute of Oceanology of RAS, 36, Nakhimovsky prosp, Moscow 117997, Russia \\ ${ }^{4}$ Laboratoire d'Océanographie Physique et Spatiale, IUEM/UBO, Rue Dumont D'Urville, 29280 Plouzané, \\ France
}

(Received 12 October 2017; accepted 27 December 2017; published online 31 January 2018)

\begin{abstract}
A simple dynamical model of vortex interactions taking place near a curved boundary mimicking a circular bay is formulated and examined. An initial configuration consisting of a point vortex in the bay and of an incident point vortex moving toward the bay along the straight part of the boundary is considered. Both vortices are of equal strengths. Typical stationary regimes of the bay-bound vortex when the incident vortex is far from the bay are obtained. When the incident vortex comes near the bay, its interaction with the bay-bound one may result in irregular motion of both vortices. Typical outcomes of the interaction are established to be (i) the incident vortex passes over the bay without forcing the bay-bound vortex to leave the bay; (ii) the incident vortex becomes entrapped within the bay, whereas the bay-bound vortex leaves it; (iii) both vortices leave the bay shortly after the interaction as separate vortices or as a bound leap-frogging pair; (iv) both vortices exhibit convoluted dynamics being entrapped for a considerable time within the bay, but in the end either one or both of the vortices leave the bay. The model might be useful in getting some insight into typical regimes of eddy dynamics near curved boundaries in the context of ocean studies provided the eddies remain coherent during the interaction. Published by AIP Publishing. https://doi.org/10.1063/1.5009117
\end{abstract}

\section{INTRODUCTION}

Many observations have revealed the presence of convoluted vortex dynamics occurring near curved coastlines in the ocean. ${ }^{1-3}$ The vortex dynamics, in this case, is governed not only by the vortices interacting with each other but also by the shape of the coastline. If the coastline can ensure the appearance of stagnation zones, vortices coming from afar might become trapped in these. On the other hand, curved coastline can be responsible for the emergence of coherent vortices by shedding vorticity from its pointed corners. ${ }^{4,5}$ Both these mechanisms can lead to the emergence of a stationary vortex inside the stagnation zone established by the curved coastline.

In this work, we are interested in studying the interaction between such a stationary vortex already situated within an enclosed coastline with an incident vortex. The model we employ is the simplest point-vortex model in a domain with a boundary mimicking a circular bay. Point-vortex models, despite offering only qualitative insights, can be useful for many applications including the study of vortex dynamics in the ocean. ${ }^{2,6-9}$ The problem of vorticity generation and the emergence of coherent vortices are not considered in the present work; instead, we assume that the vortices already exist

\footnotetext{
a)Electronic mail: ryzhovea@poi.dvo.ru

b)Electronic mail: kvkoshel@poi.dvo.ru

c)Electronic mail: sokolovskiy@iwp.ru

d)Electronic mail: xcarton@univ-brest.fr
}

before they start interacting, and continue existing after the interaction, either individually or in bound states.

Point-vortex models exhibit remarkable correspondence with more complex models of distributed vortices. ${ }^{5,10-15}$ Interacting small vorticity patches follow almost similar evolutions in the corresponding point-vortex models. Conversely, if the vorticity patches get less coherent and localized, their dynamics starts being very complex featuring spatial vorticity redistribution which results in the emergence of new vortex structures. Models of distributed vortices moving in complex bounded domains have, for example, been studied in papers. ${ }^{5,11,16-23}$

The outline of the paper is as follows. First, a model of two point vortices interacting near a circular coast-like boundary is formulated by taking advantage of the Kirchhoff-Routh stream-function. ${ }^{24}$ Stationary regimes of the bay-bound vortex are studied. Stagnation points and corresponding phase portraits are obtained. Then, a detailed examination of the dynamical regimes ensuing from the interaction between the bay-bound vortex and the incident vortex is presented. Typical regimes of the interaction are enumerated and qualitatively assessed. Evidence that the interaction is inherently irregular (meaning engendering irregular vortex trajectories) is presented in the last part of the paper.

\section{PROBLEM FORMULATION}

One can map a straight line with a circular cavity of a radius $R$ into the upper half-plane by making use of the 


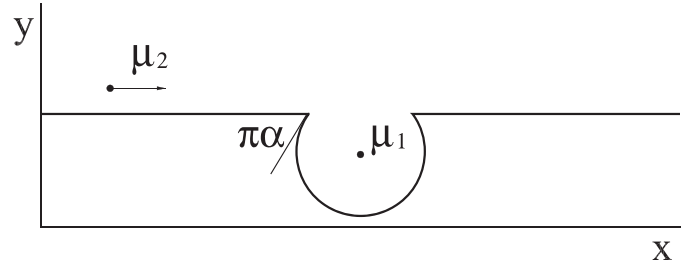

FIG. 1. The sketch of the flow with two point vortices near a curved boundary with a circular cavity. The point vortex with strength $\mu_{1}$ is initialized within the cavity, while the second point vortex with strength $\mu_{2}$ comes from infinity because of the wall-induced velocity. The multiplier $\alpha$ characterizes the "depth" or "openness" of the cavity.

mapping

$\zeta=b \frac{1}{1-\gamma \xi_{2}}, \xi_{2}=\xi_{1}^{\frac{1}{2-\alpha}}, \xi_{1}=\frac{z-a}{z+a}, \gamma=\left\{\begin{array}{l}1, \geq 0, \\ e^{2 i \pi /(2-\alpha)}, y<0,\end{array}\right.$ where $a=R \sin (\pi \alpha), b=2 a /(2-\alpha), \pi \alpha$ is the characteristic angle of the cavity (see Fig. 1), $\gamma$ determines the corresponding branch of the mapping, $z=x+i y$ corresponds to the physical domain, and $\zeta=\xi+i \eta$-mapped upper-plane domain.

We are interested in studying the dynamics of two point vortices in the original domain. Such a domain would serve as the simplest approximation of a shore with a pronounced circular bay. A point vortex with strength $\mu_{1}$ and complex coordinate $z_{1}=x_{1}+i y_{1}$ is initially situated within the bay and would remain inside the bay in the absence of perturbations. Another point vortex of strength $\mu_{2}$ and complex coordinate $z_{2}=x_{2}+i y_{2}$ is initially located near the straight boundary far from the bay (see the sketch of the flow in Fig. 1). The trajectories of the point vortices in the original domain are then governed by the Kirchhoff-Routh stream-function. ${ }^{24,25}$ Hence, the trajectories of the vortices can be figured out by means of integrating numerically the equations

$$
\begin{aligned}
& \frac{d z_{1}^{*}}{d t}=\left.\frac{d \zeta}{d z}\right|_{z=z_{1}}\left(\frac{i \mu_{1}}{\zeta\left(z_{1}\right)-\zeta^{*}\left(z_{1}\right)}-\frac{i \mu_{2}}{\zeta\left(z_{1}\right)-\zeta\left(z_{2}\right)}+\frac{i \mu_{2}}{\zeta\left(z_{1}\right)-\zeta^{*}\left(z_{2}\right)}\right)-\frac{i \mu_{1}}{2} \frac{\left.\frac{d^{2} \zeta}{d z^{2}}\right|_{z=z_{1}}}{\left.\frac{d \zeta}{d z}\right|_{z=z_{1}}} \\
& \frac{d z_{2}^{*}}{d t}=\left.\frac{d \zeta}{d z}\right|_{z=z_{2}}\left(\frac{i \mu_{2}}{\zeta\left(z_{2}\right)-\zeta^{*}\left(z_{2}\right)}-\frac{i \mu_{1}}{\zeta\left(z_{2}\right)-\zeta\left(z_{1}\right)}+\frac{i \mu_{1}}{\zeta\left(z_{2}\right)-\zeta^{*}\left(z_{1}\right)}\right)-\frac{i \mu_{2}}{2} \frac{\left.\frac{d^{2} \zeta}{d z^{2}}\right|_{z=z_{2}}}{\left.\frac{d \zeta}{d z}\right|_{z=z_{2}}}
\end{aligned}
$$

where * stands for complex conjugation. The terms with second derivatives of the domain mapping are due to the correction established by Lin, ${ }^{24}$ which ensure that the strengths of the vortices remain constant after the conformal mapping.

Since the model features a large number of parameters, we focus our attention on the case of equal strength vortices ( $\mu_{1} \equiv \mu_{2} \equiv \mu$ ), and we set the radius of the cavity to $R \equiv 1$. Despite the apparent simplification, the model still exhibits a large number of dynamical regimes of the vortices to be taken into account in detail. To limit these, only a specific initial vortex configuration is studied. Let the vortex with strength $\mu$ be enclosed in the cavity, whereas the other vortex with the same strength $\mu$ moves from afar toward the cavity along the boundary. Far from the cavity, the second vortex moves under the influence of its image vortex. ${ }^{26}$ Therefore, the second vortex moves uniformly and rectilinear along the boundary until it reaches the proximity of the cavity. Its dynamics is then impacted greatly by the presence of the cavity and of the cavity-bound vortex enclosed in it. The sketch of the flow is depicted in Fig. 1.

The cavity-bound vortex, on the other hand, is initially located inside the cavity. In the absence of perturbation, i.e., when the incident vortex is far from the cavity, the cavity-bound vortex remains near its initial position. This is ensured by choosing the cavity-bound vortex's initial position close to the elliptic critical point of the corresponding steady one-vortex system. ${ }^{27}$ The cavity-bound vortex remains near the elliptic critical point until the incident vortex comes close enough to start exerting a significant influence on the cavitybound vortex. Then the dynamics of both vortices becomes convoluted and unpredictable. This lack of predictability follows from the fact that the interaction of these two vortices is generally irregular in the bounded domain. Irregular motion, in this case, means that two initially nearby phase trajectories exponentially diverge from each other. ${ }^{28-30}$ Many studies show that domains with curved boundaries produce complex motion of single vortices and passive scalars. $^{31-35}$

\section{A SINGLE VORTEX INSIDE THE CAVITY}

First, we need to look for stagnation points of the flow with only one vortex moving near a straight boundary with a circular cavity. To this purpose, the incident vortex is removed. Then, the governing equation for the cavity-bound vortex reduces to $^{27}$

$$
\frac{d z_{0}^{*}}{d t}=\left.\frac{d \zeta}{d z}\right|_{z=z_{0}}\left(\frac{i \mu}{\zeta\left(z_{0}\right)-\zeta^{*}\left(z_{0}\right)}\right)-\frac{i \mu}{2} \frac{\left.\frac{d^{2} \zeta}{d z^{2}}\right|_{z=z_{0}}}{\left.\frac{d \zeta}{d z}\right|_{z=z_{0}}}
$$

where $z_{0}=x_{0}+i y_{0}$ is the complex coordinate of the vortex for the case of single vortex dynamics. 


\section{A. An analysis of critical points}

Though we could not find closed analytical expressions for the critical points following from (3), we can still present a qualitative analysis of the number of critical points. Equating the right-hand term in (3) to zero and noticing that all the critical points lie at the $y$-axis, i.e., $x_{0}=0$, one obtains

$$
\begin{gathered}
\left.\frac{\xi(\xi-b)}{z^{2}-a^{2}}\right|_{z=i y_{0}}\left(\frac{1}{2 i \eta\left(i y_{0}\right)}\right)-\left.\frac{1}{2} \frac{2 \xi-b-2 z}{z^{2}-a^{2}}\right|_{z=i y_{0}} \\
=\frac{-\frac{b^{2}}{4}+\eta^{2}\left(i y_{0}\right)-2 y_{0} \eta\left(i y_{0}\right)}{2 i \eta\left(i y_{0}\right)\left(y_{0}^{2}+a^{2}\right)}=0
\end{gathered}
$$

where the derivatives $\frac{d \zeta}{d z}=\frac{\xi(\xi-b)}{z^{2}-a^{2}}, \frac{d^{2} \zeta}{d z^{2}} / \frac{d \zeta}{d z}=\frac{2 \xi-b-2 z}{z^{2}-a^{2}}$ are already taken into account, and $\eta$ is the imaginary part of the mapping $\zeta$ defined earlier. Also, one can notice that the $y$-axis is mapped into the axis $\xi=b / 2$. Then the vertical coordinates for the critical points ensue

$$
\eta\left(i y_{0}\right)=y_{0}+\sqrt{y_{0}^{2}+\frac{b^{2}}{4}}=-\frac{b}{2} \cot \left(\frac{\arctan \left(y_{0} / a\right) \pm \frac{\pi}{2}}{2-\alpha}\right),
$$

hence

$$
\begin{aligned}
f_{1} & =f_{2}, \\
f_{1}\left(y_{0} /\left(\frac{b}{2}\right)\right) & =y_{0} /\left(\frac{b}{2}\right)+\sqrt{y_{0}^{2} /\left(\frac{b}{2}\right)^{2}+1} \\
f_{2}\left(y_{0} /\left(\frac{b}{2}\right)\right) & =-\cot \left(\frac{\arctan \left(\left(y_{0} /\left(\frac{b}{2}\right)\right) \frac{1}{2-\alpha}\right) \pm \frac{\pi}{2}}{2-\alpha}\right),
\end{aligned}
$$

where the sign of $\pm \frac{\pi}{2}$ is the same as the sign of $y_{0}$. The term $\eta\left(i y_{0}\right)$ is always non-negative; therefore, the second branch of the solution to (4) is inapplicable. Figure 2 shows the values of the left-hand term (curve 1) and the right-hand term (curve 2 $\alpha=0.1$, curve $3-\alpha=0.2304$, curve $4-\alpha=0.3$ ) of (6) depending on $y_{0} / \frac{b}{2}$. Equation (6) has up to two zeroes corresponding

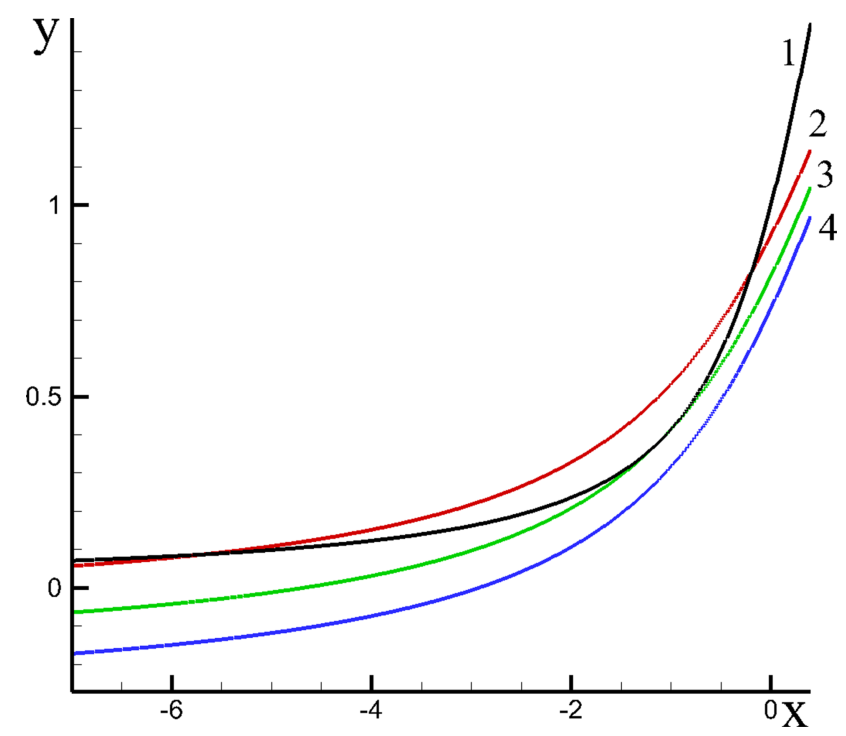

FIG. 2. $x$-axis $-y_{0} / \frac{b}{2} ; y$-axis is the values of $f_{1}$ (curve 1 ) and $f_{2}$ [curve 2- $\alpha$ $=0.1$ (two intersections with curve 1 correspond to two critical points), curve $3-\alpha=0.2304$ (one intersection with curve 1 corresponds to one critical point), curve 4- $\alpha=0.3$ (no intersections with curve 1-no critical points)] of Eq. (6). to elliptic and hyperbolic critical points. Elliptic critical points are stable critical points, near which the phase trajectories of the system are ellipses. Hyperbolic critical points are unstable critical points, near which the phase trajectories of the system are hyperboles. Two solutions are possible for small values of $\alpha$ (see two intersections between curve 1 and curve 2 for $\alpha=0.1$ ). When $\alpha \sim 0.2304$, the critical points coalesce (see one tangency point of curve 1 and curve 3 for $\alpha=0.2304$ ); as $\alpha$ increases the critical points vanish (curve 1 and curve 4 for $\alpha=0.3$ do not intersect).

\section{B. Phase portraits}

The phase portrait of the single vortex system depends on the parameter $\alpha$ that determines the "depth" of the cavity. The influence of the "depth" of the cavity is straightforward; the deeper the cavity, the larger the measure of periodic trajectories inside it. Figure 3 depicts typical phase portraits of the vortex motion for different depths of the cavity. Figure 3(a) shows the phase portrait near a degenerate case, when the stagnation point has just appeared in the system. The corresponding value of the parameter $\alpha=0.2304$. As the parameter $\alpha$ increases, the depth of the cavity decreases, and no stagnation point occurs in the flow.

As the parameter $\alpha$ decreases, the measure of periodic vortex trajectories increases [see examples presented in Figs. 3(b) and 3(c) for $\alpha=0.2$ and 0.1, respectively]. The bold blue line indicates the separatrix of the flow, the line dividing different types of motion. The bold black line marks the boundary of the domain. The elliptic stagnation point is designated by a bold dot. It is worth noting that the shape of the trajectories in the phase portraits of the steady-state dynamics of a single point vortex is independent of the vortex's strength $\mu$. The strength affects only the speed of the vortex translation through the flow.

Judging by the phase portraits, one can say that the cavitybound vortex is always stable within the region of periodic phase trajectories, and it is unstable if positioned exactly at the hyperbolic point. When unstable [see Fig. 3(a)], any small perturbation to the vortex's initial position forces it to leave the cavity. When stable [for example, see Fig. 3(c)], even finiteamplitude perturbations to the vortex's initial position do not cause the vortex escaping the cavity.

\section{INTERACTION OF AN ALONG-SHORE PROPAGATING VORTEX WITH A VORTEX IN THE BAY}

Next, after establishing typical dynamical patterns of the single vortex dynamics, we initialize a second point vortex coming toward the cavity from afar along the boundary due to a wall-induced velocity. The wall-induced propagation of the second vortex proceeds almost unperturbed until it reaches the immediate proximity of the cavity, where it starts interacting with the cavity-bound vortex. Their interaction is irregular in its nature and produces generally unpredictable outcomes. The possible outcomes are as follows.

First, the incident (along-shore propagating) vortex may simply leap across the cavity's orifice and continue its wall-induced motion along the boundary. This behavior is 


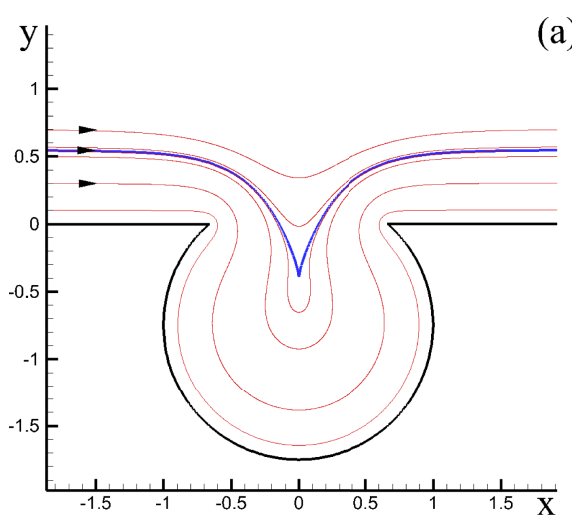

(a)
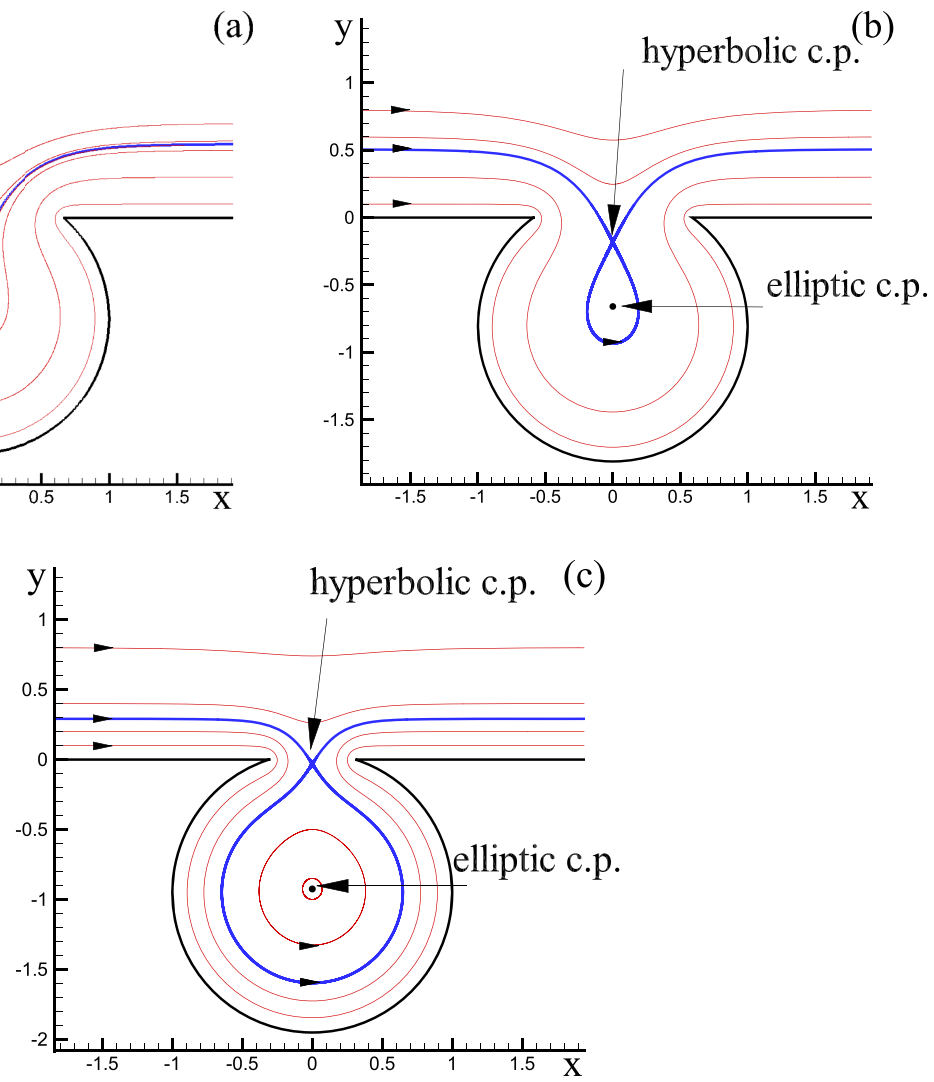

FIG. 3. Examples of the phase portraits of the steady-state trajectories of a single point vortex initialized inside the cavity for different values of parameter $\alpha$ : (a) 0.2304 ; (b) 0.2 ; (c) 0.1 . The separatrix demarcating the different types of motion is the bold blue line. The corresponding critical points are marked in the figures. tantamount to the case of a single vortex leaping across a gap in a straight boundary. ${ }^{11}$ Thus, the incident vortex influence on the cavity-bound vortex is deemed negligible. A similar outcome is observed when the incident vortex moves fast enough and close enough to the boundary so that it always remains close to the boundary even when it enters the cavity. If the cavity-bound vortex is located relatively far from the boundary, it may remain relatively unperturbed by the incident vortex.

Second, the incident and cavity-bound vortices may swap roles, as the incident vortex becomes cavity bound, whereas the initially cavity-bound vortex leaves the cavity and moves away from it, along the boundary, due to the wall-induced velocity.

Third, the incident and cavity-bound vortices may both leave the cavity and either produce a leap-frogging pair ${ }^{36-39}$ that moves off the cavity due to the wall-induced velocity or become two separate along-shore propagating vortices at a significant distance from each other.

Fourth, after the incident vortex gets into the cavity, both vortices may form a bound vortex structure that remains in the cavity for a while. However, this structure cannot remain in the cavity forever because of the irregularity of the interaction. Eventually, the bound structure either breaks or leaves the cavity as a leap-frogging pair.

Since the vortex interaction under study is irregular, an approach taking into account many initial vortex positions seems most appropriate and fruitful. The initial positions of the cavity-bound and incident vortices are $\left(x_{1}(0), y_{1}(0)\right)$ and $\left(x_{2}(0), y_{2}(0)\right)$, respectively. The initial position of the incident vortex on the $y$-axis is taken in the interval $0<y_{2}(0)$ $\leq 2$ while its initial $x$-coordinate is fixed at $x_{2}(0) \equiv-5$. The initial position of the cavity-bound vortex is fixed for every $y_{2}(0)$ at a point inside the recirculation zone including the critical points (see Fig. 3). Then, the governing Eqs. (2) are numerically integrated for $5 \times 10^{4}$ incident vortex's initial positions $y_{2}(0)$, distributed uniformly within the interval $0<y_{2}(0) \leq 2$. The calculation continues until either both vortices cross the vertical line $x=20$ (crossing this line, the vortices are assumed not to be possibly able to return to the cavity) or the model time reaches $t=150$ (a typical time for the incident vortex to cross the control line when there is no cavity-bound vortex is $t \sim 20$ ). The sought-after characteristic is the time each vortex takes to cross the control line. The time distribution, further referred to as the escape time, shows which initial $y$-coordinates of the incident vortex result in predominately regular or irregular motion.

It is worth noting that the system is also sensitive to the choice of the initial position $x_{2}(0)$, but only when $x_{2}(0)$ is taken in the immediate vicinity of the bay. When $x_{2}(0)$ is chosen far from the bay, the incident vortex moves along a straight line parallel to the straight boundary such that $x_{2}=$ const before it comes close to the cavity. Therefore, we intentionally take $x_{2}(0)=-5$, which is far enough from the bay in order to filter out its influence.

\section{A. Unstable cavity-bound vortex}

Let us start by considering the case of the initially unstable cavity-bound vortex. The phase portrait of the singlevortex dynamics is shown in Fig. 3(a). Figure 4(a) illustrates the escape time distribution depending on the incident vortex's initial $y$-position for the initial vortex configuration: the cavity-bound vortex is situated at the hyperbolic point 

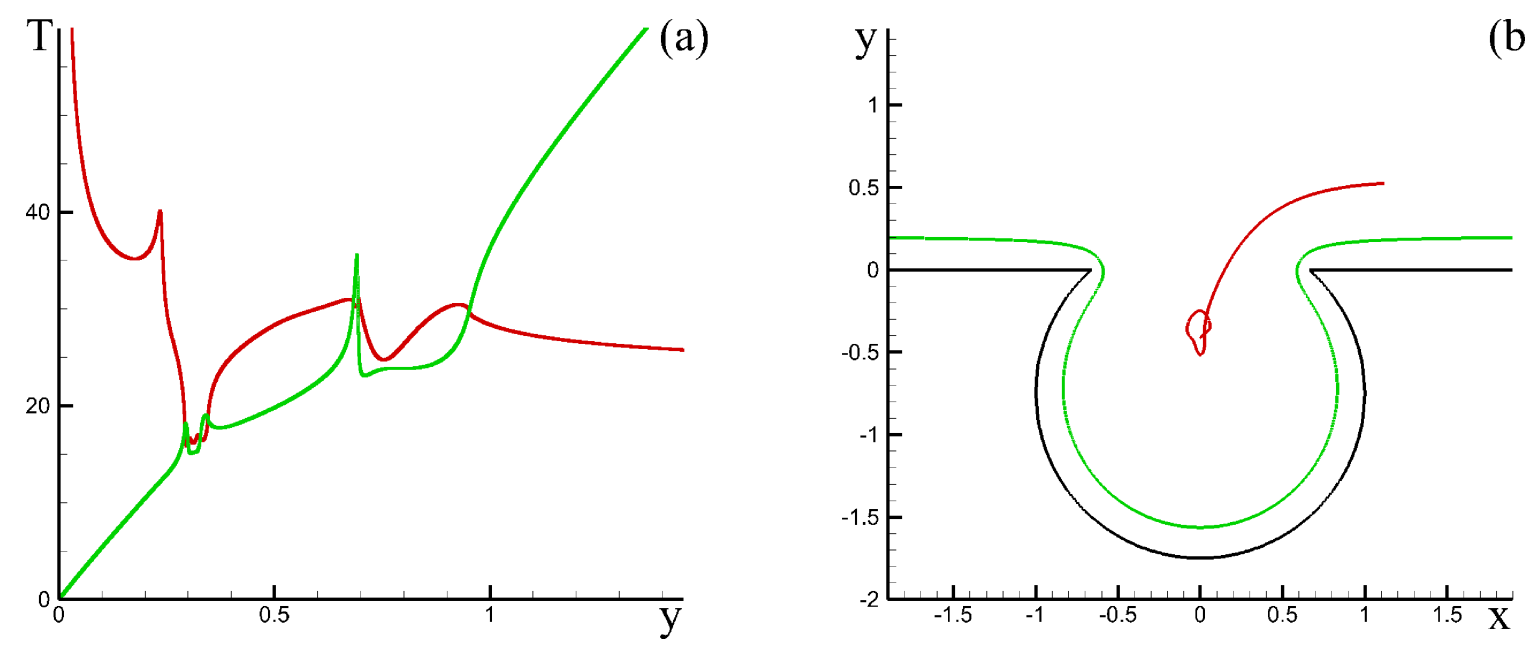

(b)
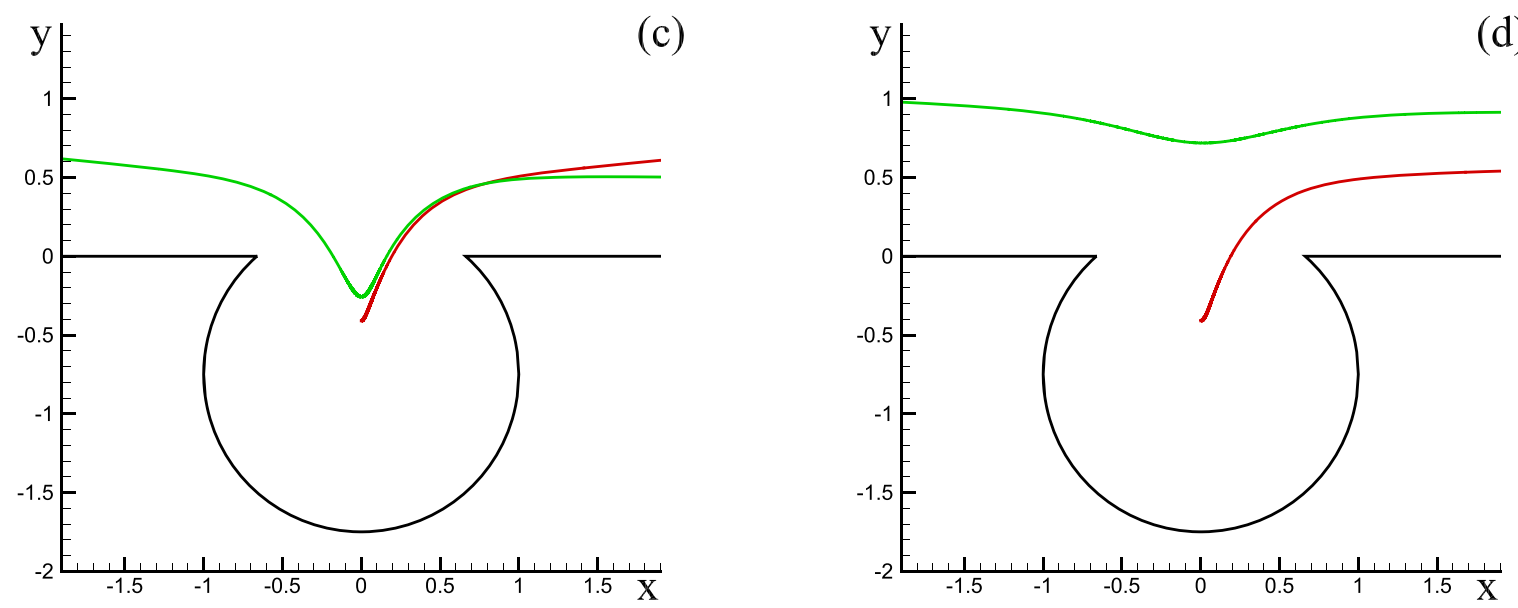

FIG. 4. (a) Escape time distributions for the cavity-bound vortex (red line) and the incident vortex (green line) as the cavity-bound vortex is initialized at the corresponding critical point of the phase portrait shown in Fig. 3 for $\alpha=0.2304$, hyperbolic critical point at $y_{1}(0)=-0.41$. Examples of trajectories for the incident vortex's initial $y$-position (b) $y_{2}(0)=0.2$, (c) $y_{2}(0)=0.7$, (d) $y_{2}(0)=1.1$.

$x_{1}(0)=0, y_{1}(0)=-0.41$; the incident vortex lies at $x_{2}(0)=-5$. The red and green curves correspond to the cavity-bound and incident vortices, respectively.

Since the curves indicate that the vortices behave highly irregularly, only a rather rough classification of the regimes is possible. One can point out three typical regimes of motion for the incident vortex (green line). The first one corresponds to the smooth part from $y_{2}(0)=0$ until approximately $y_{2}(0) \sim 0.3$. In this case, the incident vortex gets inside the cavity but moves fast and close enough to the boundary, which allows it not to be entangled with the cavity-bound vortex. Hence, the incident vortex simply passes the cavity as if it was just a straight line. An example of the corresponding vortex trajectories for the initial incident vortex position $y_{0}(2)=0.2$ is shown in Fig. 4(b), where the incident vortex trajectory is plotted in green and the cavity-bound one is plotted in red. In this regime, the incident vortex has a much smaller escape time than that of the cavitybound vortex. The cavity-bound vortex (red line), on the other hand, experiences small perturbations induced by the incident vortex and leaves the cavity following the incident vortex.

The next regime for approximately $0.3 \lesssim y_{2}(0) \lesssim 1$ is the most irregular one resulting in generally unpredictable dynamics. In this case, the incident vortex impacts directly the cavity-bound vortex leading both vortices to leave the cavity [examples of trajectories are shown in Fig. 4(c)].

The corresponding phase portrait for the cavity-bound vortex is shown in Fig. 3(a). It features only one critical point where the cavity-bound vortex is stationary. Any small perturbation makes the vortex leave the cavity. Therefore, when the incident vortex comes close to the cavity, it always displaces the cavity-bound vortex into the region of unbounded phase trajectories. Thus, there is only one possible outcome of this vortex interaction: the vortices always leave the cavity by either forming a leap-frogging pair or by moving separately along the boundary.

The last regime occurs for values of $y_{2}(0) \gtrsim 1$. An example of trajectories is shown in Fig. 4(d). In this case, the incident vortex moves at a significant distance from the boundary, which results in a slower wall-induced velocity (therefore, significantly increasing the incident vortex escape time). This, in turn, ensures that the incident vortex does not get inside the cavity, jumping over the orifice instead. Nevertheless, the incident vortex simply passing near the cavity perturbs the 
cavity-bound vortex forcing it to leave the cavity immediately after the incident vortex.

Another case of an unstable cavity-bound vortex incurs from the initial cases featuring two critical points, one of which is hyperbolic, i.e., unstable. When the cavity-bound vortex is positioned at the hyperbolic critical point, the escape-time distributions evince features similar to the case considered earlier, when both vortices leave the cavity because of the interaction. However, since there is a sizeable recirculation zone initially, after the incident vortex affects the cavity-bound vortex, it may, instead of leaving the cavity, get inside the recirculation zone and remain trapped near the elliptic critical point. Figure 5(a) shows the corresponding escape time distributions for $\alpha=0.2$, when the cavity-bound vortex is positioned at the hyperbolic point $y_{1}(0)=-0.182$. When the vortices leave the cavity, their escape time distributions are similar to the case with no recirculation zone in the initial state. Also, regimes when one of the vortices is trapped, whereas the other leaves the cavity, start manifesting themselves. Figure 5(b) depicts an example $\left(y_{2}(0)=0.575\right)$ of vortex trajectories when the incident vortex remains trapped, whereas the initially cavity-bound vortex leaves the cavity.

\section{B. Stable cavity-bound vortex}

We next move on to the initial states when the cavitybound vortex is positioned at an elliptic critical point like the ones shown in Figs. 3(b) and 3(c). When positioned initially at the elliptic critical point, the cavity-bound vortex remains trapped inside the cavity, even for small perturbations, due to the interaction with the incident vortex.

First, the case of a relatively small periodic motion region is examined. The initial state corresponds to that of $3 b$ as $\alpha=0.2$; therefore, the initial cavity-bound vortex's position is at the elliptic critical point $x_{1}(0)=0, y_{1}(0)=-0.6595$. The initial coordinates of the incident vortex are the same as in the previous numerical experiment $x_{2}(0)=-5,0<y_{2}(0) \leq$ 2. The escape time distribution is illustrated in Fig. 6(a). A stark increase in complexity of the escape time distributions compared to the previous case is apparent. The main difference is the emergence of several infinite (or at least very long) escape times, which means that entrapping of one of the vortices becomes possible. In this case, a regime of unambiguously regular dynamics can be singled out, namely, the one for $y_{2}(0)$ $\lesssim 0.25$. This regime is similar to that considered earlier: the incident vortex moves fast and close enough to the boundary, allowing it to pass over the cavity without being entangled with the cavity-bound vortex. The only difference with the case analyzed earlier is that the cavity-bound vortex remains trapped inside the cavity.

As $0.25 \lesssim y_{2}(0) \lesssim y^{*}\left(y^{*}\right.$ is some relatively large value when the vortex interaction becomes weak), the dynamics of the vortices becomes highly unpredictable with many transient effects. An example of vortex trajectories when both vortices leave the cavity is shown in Fig. 6(b) for $y_{2}(0)=0.54$. A small change in the initial value of $y_{2}(0)=0.55$ results in the cavitybased vortex sustaining the perturbation and remaining trapped inside the cavity [see Fig. 6(c)]. Figure 6(d) $\left(y_{2}(0)=0.867\right)$, on the other hand, exemplifies how the incident vortex can be entrapped instead of the cavity-bound vortex. The entrapping may happen because the incident vortex is pulled into the cavity owing to the established bond with the cavity-bound vortex. Moreover, when $y_{2}(0) \gtrsim y^{*}$, the incident vortex jumps over the cavity's orifice and forces the cavity-bound vortex to leave the cavity at the same time because the time of the interaction increases as the incident vortex's wall-induced translation velocity decreases. Thus, although the influence of the incident vortex toward the cavity-bound one is weaker, it lasts longer.

When the cavity's orifice decreases, the range of the initial positions $y_{2}(0)$ which lead to the incident vortex jumping over the cavity and with little effect on the cavity-bound vortex, becomes large. On the other hand, if the incident vortex gets inside the cavity and interacts with the cavity-based vortex, the dynamics becomes complicated. This is because the vortices remain inside the cavity for longer times, which makes their trajectories intertwine more often. Figure 7(a) illustrates the escape time distribution for the initial state corresponding to the phase portrait shown in Fig. 3(c) $\left(\alpha=0.1, y_{1}(0)=-0.925\right)$.
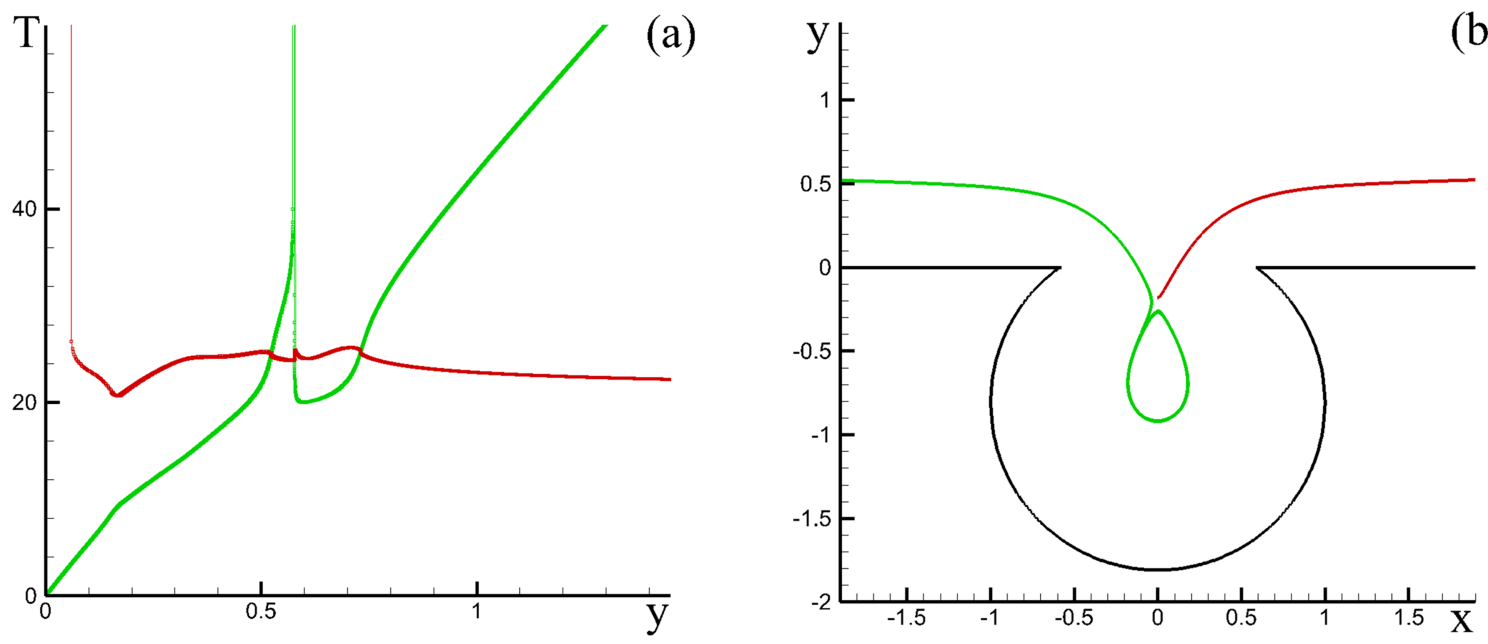

(b)

FIG. 5. (a) The same as in Fig. 4(a) for $\alpha=0.2$, and the cavity-bound vortex is initially positioned at the hyperbolic critical point at $y_{2}(0)=-0.18$; (b) an example of vortex trajectories for $y_{2}(0)=0.575$ as the incident vortex becomes trapped, whereas the cavity-bound vortex leaves the cavity. 

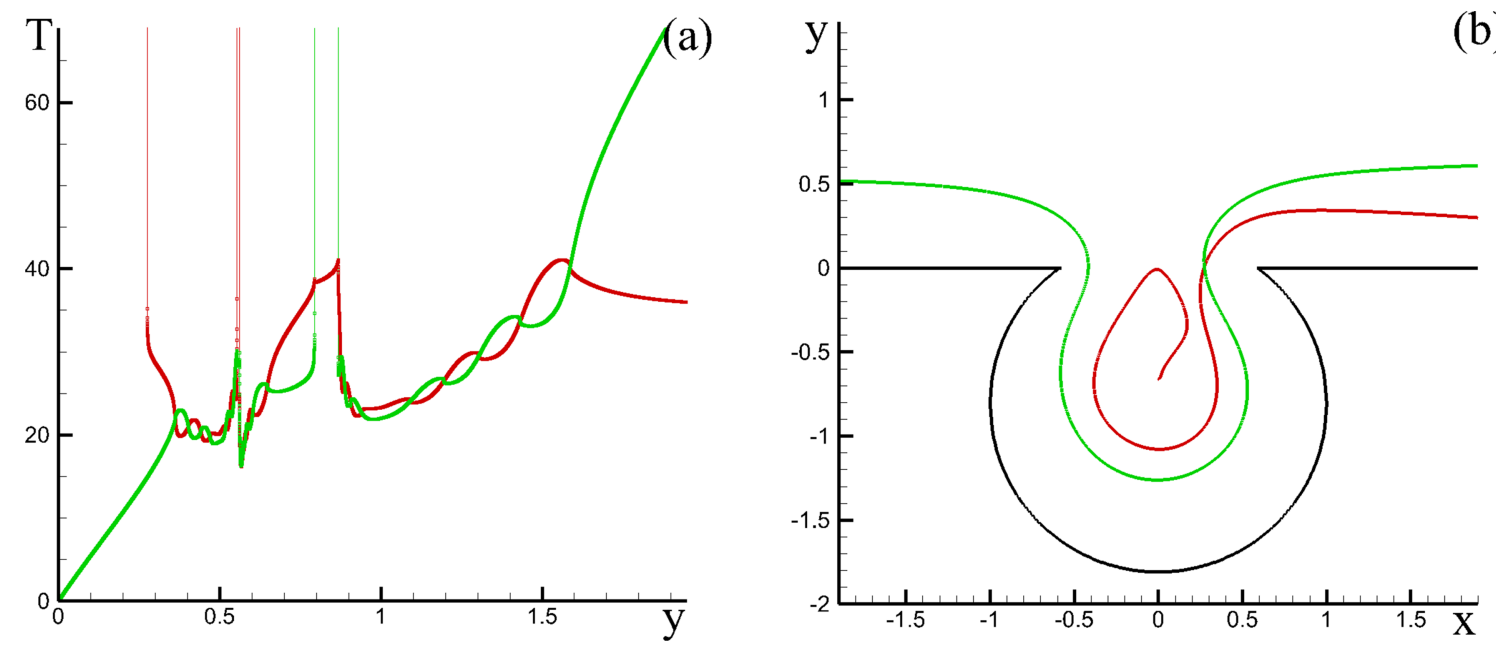

(b)
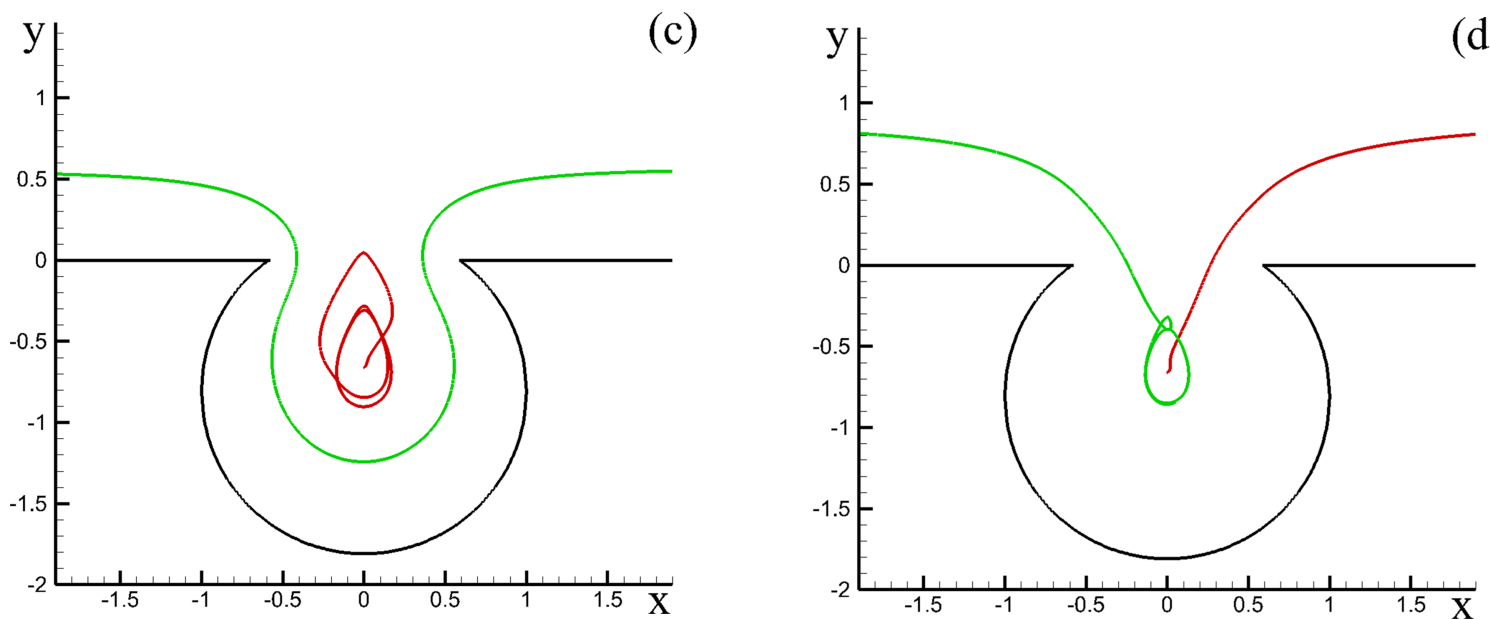

(d)

FIG. 6. (a) The same as in Fig. 4(a) for $\alpha=0.2, y_{1}(0)=-0.6595$. Examples of vortex trajectories for (b) $y_{2}(0)=0.54$, (c) $y_{2}(0)=0.55$, (d) $y_{2}(0)=0.867$.

The regular regimes, when the incident vortex moves either very close to the boundary or very far from it, are similar to the corresponding regimes of the first case addressed earlier (see Fig. 4). The only difference is that the cavitybound vortex remains trapped inside the cavity. However, if the incident vortex gets inside the cavity and impacts directly the cavity-bound one, the ensuing dynamics becomes irregular.

Regimes with permanent entrapping of one of the vortices appear as in the previous case, but there also occur regimes with finite-time entrapping of both vortices. As the initial value of $y_{2}$ changes, all these regimes alter irregularly. Figures $7(b)$ and $7(c)$ for $y_{2}(0)=0.411$ and $y_{2}(0)=0.41$, respectively, evince the irregular nature of the vortex trajectories. Both figures correspond to the case of finite-time entrapping of both vortices, but in Fig. 7(b) the cavity-bound vortex escapes the cavity, whereas in Fig. 7(c) it is the incident vortex, which escapes the cavity. A regime when both vortices leave the cavity almost simultaneously is also possible [see an example in Fig. 7(d) for $y_{2}(0)=0.41416$ ]. It is worth noting that there are also cases similar to the ones shown in Figs. 6(c) and 6(d), with one of the vortices escaping the cavity swiftly without spending much time inside the cavity. When $y_{2}(0)$ is relatively large, the incident vortex influence is negligible, hence the cavity-bound vortex remains trapped inside the cavity. Indeed, the influence of the incident vortex decreases as $y_{2}(0)$ increases; moreover, the cavity's orifice is small in this case, which ensures a smaller interaction time and a large stable region for the cavity-bound vortex. Another observation is that the distance from which the incident vortex can be dragged into the cavity decreases $\left(y_{2}(0) \sim 0.4\right)$ in comparison with the case of $\alpha=0.2\left(y_{2}(0) \sim 1.6\right)$ shown in Fig. 6.

\section{Phase dependence of the escape-time distributions}

Up to this section, we have considered only cases when the cavity-bound vortex is initialized at one of the critical points of the corresponding stationary phase portrait. However, if one initializes the cavity-bound vortex at an arbitrary position within the closed recirculation zone, it would dramatically change the corresponding escape-time distributions. Indeed, when initialized on a periodic phase trajectory, the cavitybound vortex has different current position for every incident vortex initial position $y_{2}(0)$, as the incident vortex comes close to the cavity. As $y_{2}(0)$ increases, the wall-induced velocity of the incident vortex, $\frac{\mu}{2 y_{2}(0)}$, decreases; therefore, the time the incident vortex needs to come near the cavity increases. Since, 

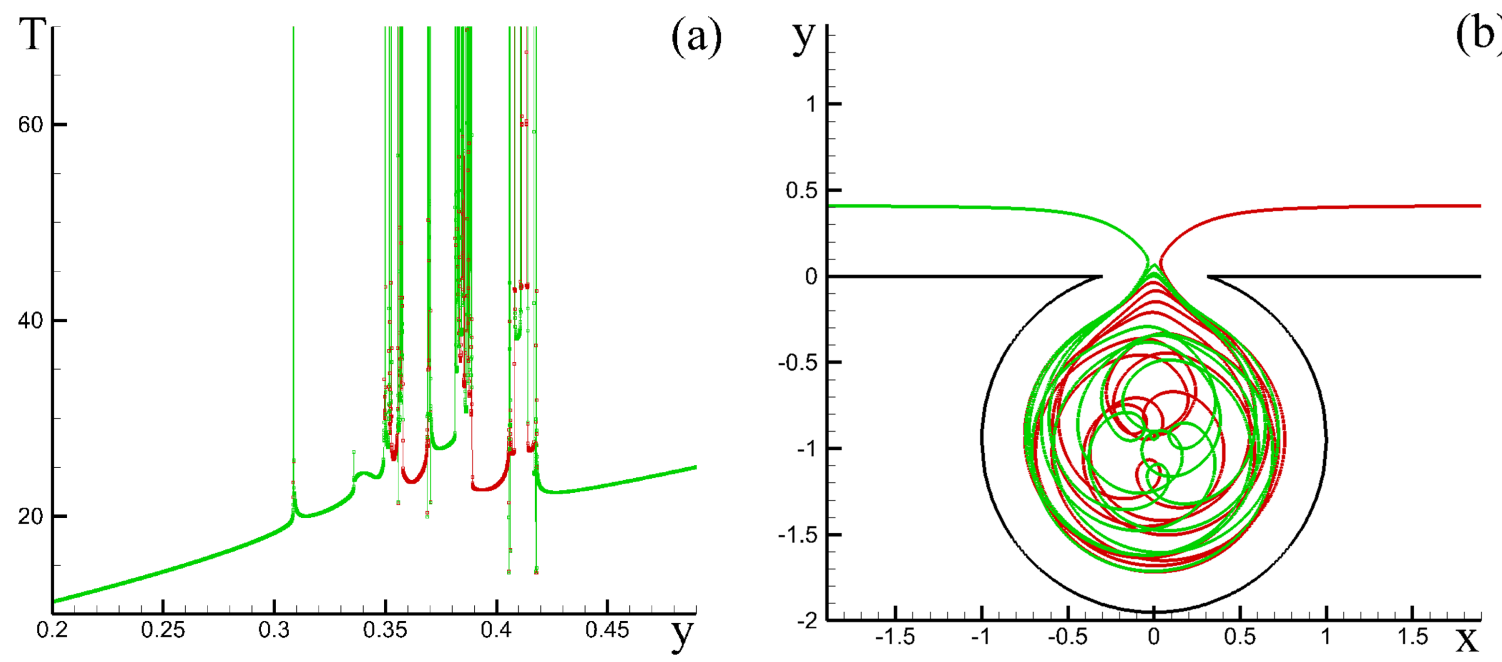

(b)
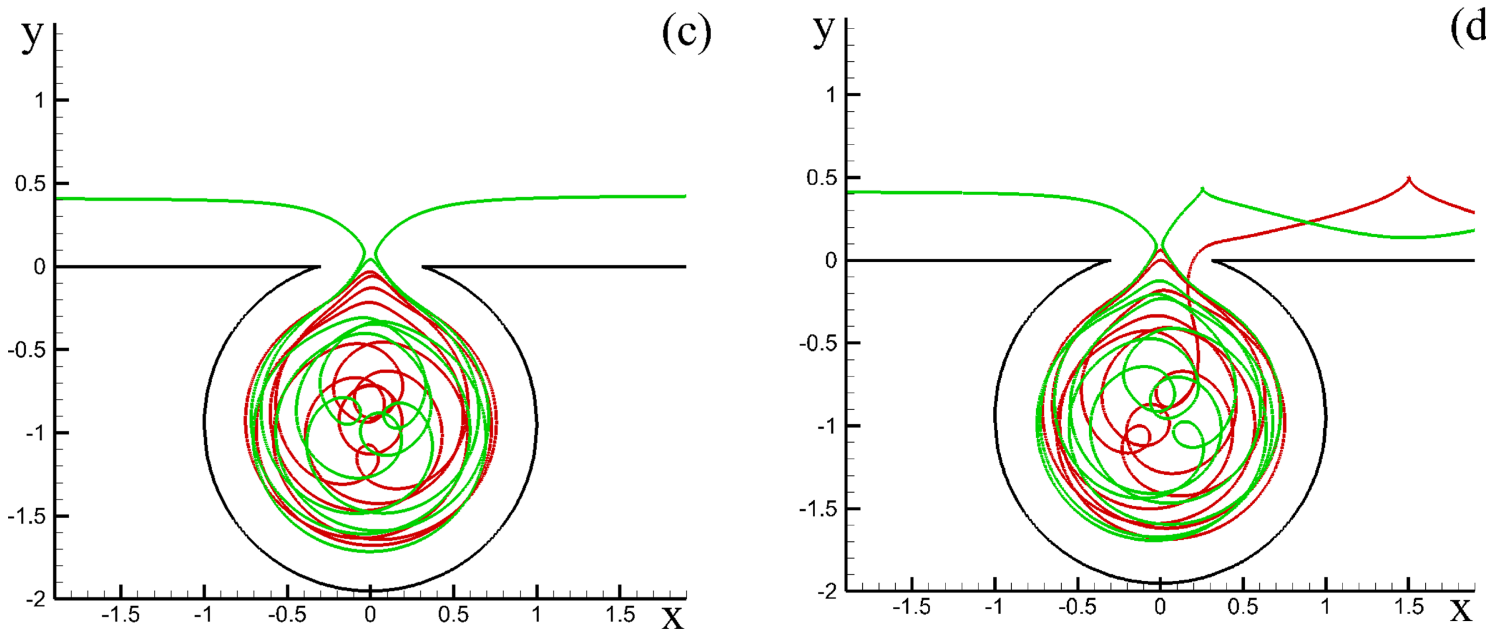

(d)

FIG. 7. (a) The same as in Fig. 4(a) for $\alpha=0.1, y_{1}(0)=-0.925$. Examples of vortex trajectories for (b) $y_{2}(0)=0.411,(\mathrm{c}) y_{2}(0)=0.41,(\mathrm{~d}) y_{2}(0)=0.41416$.

now the cavity-bound vortex is not fixed initially at a critical point, it oscillates about the corresponding critical point, so that the distance between the vortex and the orifice changes in time. Thus, now, for different $y_{2}(0)$, when the incident vortex comes near the cavity, the cavity-bound vortex can be located at different positions.

Figure 8 illustrates the escape-time distributions for the cavity-bound vortex's initial position (a) $x_{1}(0)=-0.17, y_{1}(0)$ $=-1.274$ and $(\mathrm{b}) x_{1}(0)=-0.321, y_{1}(0)=-0.725$.

The figure shows that the vortices can interact intensely. In this case, the intense interaction implies that the vortices come close enough to each other and, consequently, form a bound vortex structure. Its dynamics is generally irregular leading the bound vortex structure either to break into two separate vortices or to leave the cavity and to leap-frog along the straight boundary.

According to Fig. 8(a), one can single out two zones when the vortex interaction is intense. The first zone of the intense interaction $\left[0.2 \lesssim y_{2}(0) \lesssim 0.5\right.$ in Fig. 8 (a) and $0.25 \lesssim y_{2}(0)$ $\lesssim 0.6$ in Fig. 8(b)] corresponds to relatively small $y_{2}(0)$, leading the incident vortex to enter the cavity. The incident vortex gets inside the cavity if its trajectory lies below the stationary separatrix shown in Fig. 3(c). The upper boundary of the intense interaction zone may lie above the separatrix value, and the incident vortex can be pulled into the cavity, if it establishes a strong bond with the cavity-bound vortex located nearby. The lower boundary of the intense interaction zone lies slightly below the separatrix. The vortex dynamics in this case is very complex. Regimes with distinct dynamics alter irregularly. Such regimes are (i) the cavity-bound vortex leaving the cavity, while the incident one gets entrapped [similar trajectories as in Figs. 4(b), 6(d), and 7(b)]; (ii) the vortices form a bound vortex structure and leave the cavity [similar trajectories as in Figs. 6(c) and 7(d)].

In general, the incident vortex may get inside the cavity and have a chance to remain in it by two distinct mechanisms. First, it can get inside the cavity because it simply moves close enough to the boundary. Second, the cavity-bound vortex may force the incident one to get inside the cavity provided they are located relatively close when the incident vortex passes near the cavity's orifice.

The second zone of intense interaction [the significantly lower escape time between two peaks for $0.75 \lesssim y_{2}(0) \lesssim 0.9$ in Fig. 8(a)] results from the incident vortex passing over the cavity. This may happen if the cavity-bound vortex is located near the cavity's orifice and the incident vortex has 

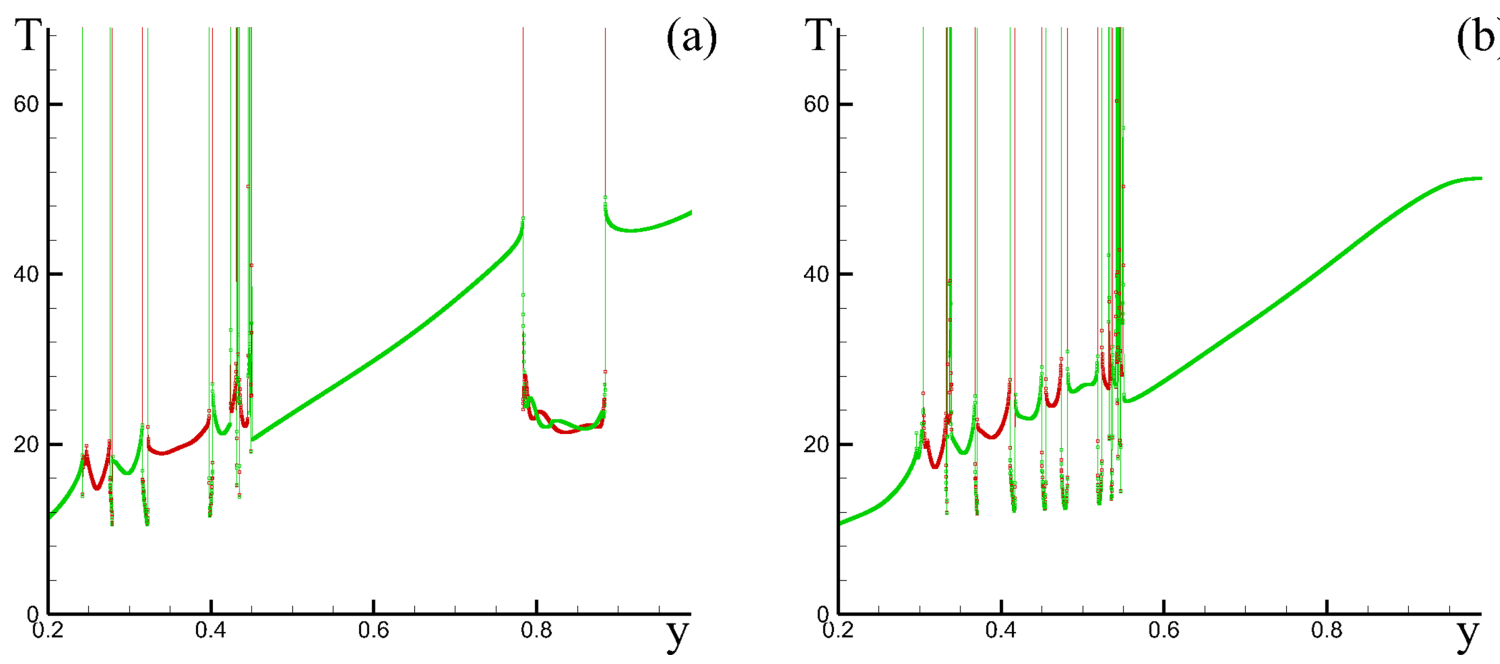

(b)

FIG. 8. Escape-time distributions for $\alpha=0.1$ for the cases, when the cavity-bound vortex is initialized on an initially periodic trajectory: (a) $x_{1}(0)=-0.17$, $y_{1}(0)=-1.274 ;$ (b) $x_{1}(0)=-0.321, y_{1}(0)=-0.725$.

already approached the cavity's orifice. In this case, the distance between the vortices is rather small, and, therefore, they interact intensely.

On the other hand, there can be no such second zone, provided the cavity-bound vortex and the incident vortex are located far from each other when the incident vortex passes near the cavity (the incident vortex is near the cavity's orifice, while the cavity-bound vortex is near the "bottom" of the cavity), and the interaction is significantly weaker [the escape-time distributions in Fig. 8(b) feature no anomalies for $\left.y_{2}(0) \gtrsim 0.6\right]$, but, nevertheless, both vortices leave the cavity [similar trajectories as in Figs. 4(c) and 4(d)]. When $y_{2}(0)$ increases further, the incident vortex passes over the cavity's orifice without exerting much influence on the cavity-bound one. This is attributed to the fact that a narrow cavity's orifice at small $\alpha \sim 0.1$ induces a large recirculation zone inside the cavity and also allows the vortices to interact only for a short time.

\section{Fluid particle advection induced by the two-vortex interaction}

Here, we briefly discuss how the considered two-vortex interaction affects the fluid particle advection in its immediate vicinity. The fact that vortices stir the neighboring fluid particles and thus induce intricate advection of these fluid particles is well-established and has been studied at length (see, for example, Refs. 8 and 40-57). Therefore, it is interesting to look into the fluid particle advection patterns induced by the interaction between the incident and cavity-bound vortices. To do this, we uniformly distribute $35 \times 10^{4}$ markers within a circle with radius $R$ that is nested into the cavity.
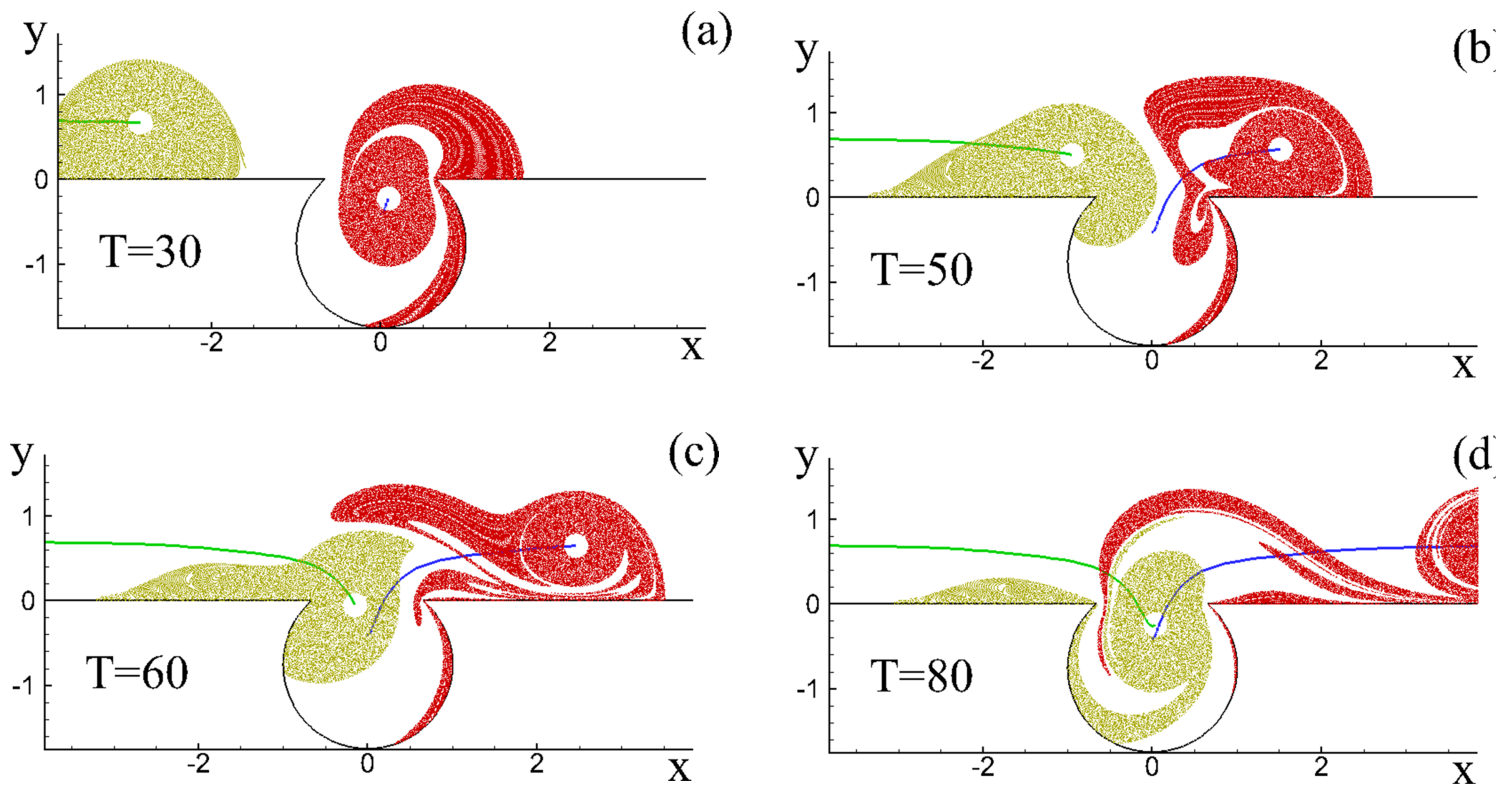

(c)

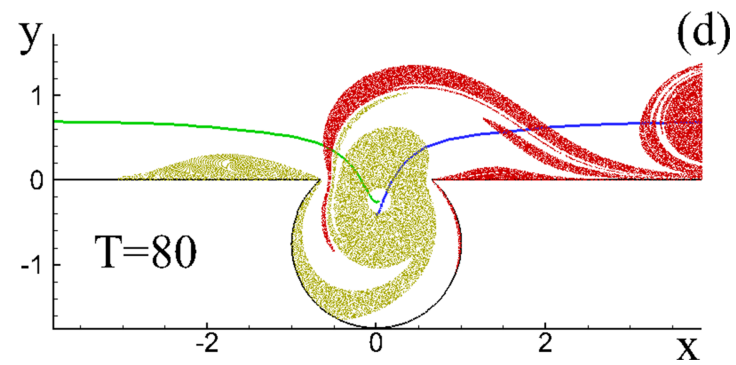

FIG. 9. The scattering of fluid particles induced by the two-vortex interaction corresponding to the vortex trajectories shown in Fig. 4(c). Consequential instant distributions of the fluid particles for the given calculation time. 


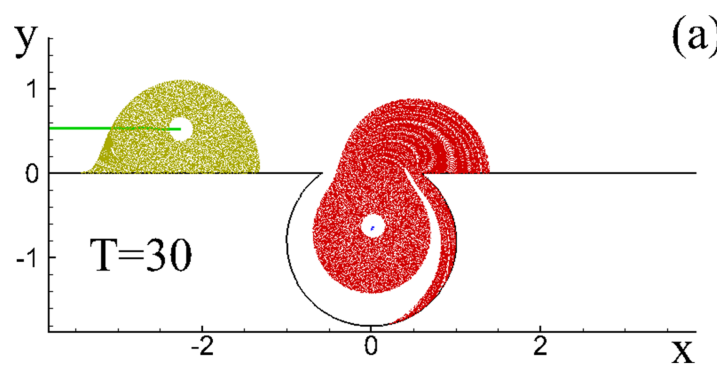

(a)
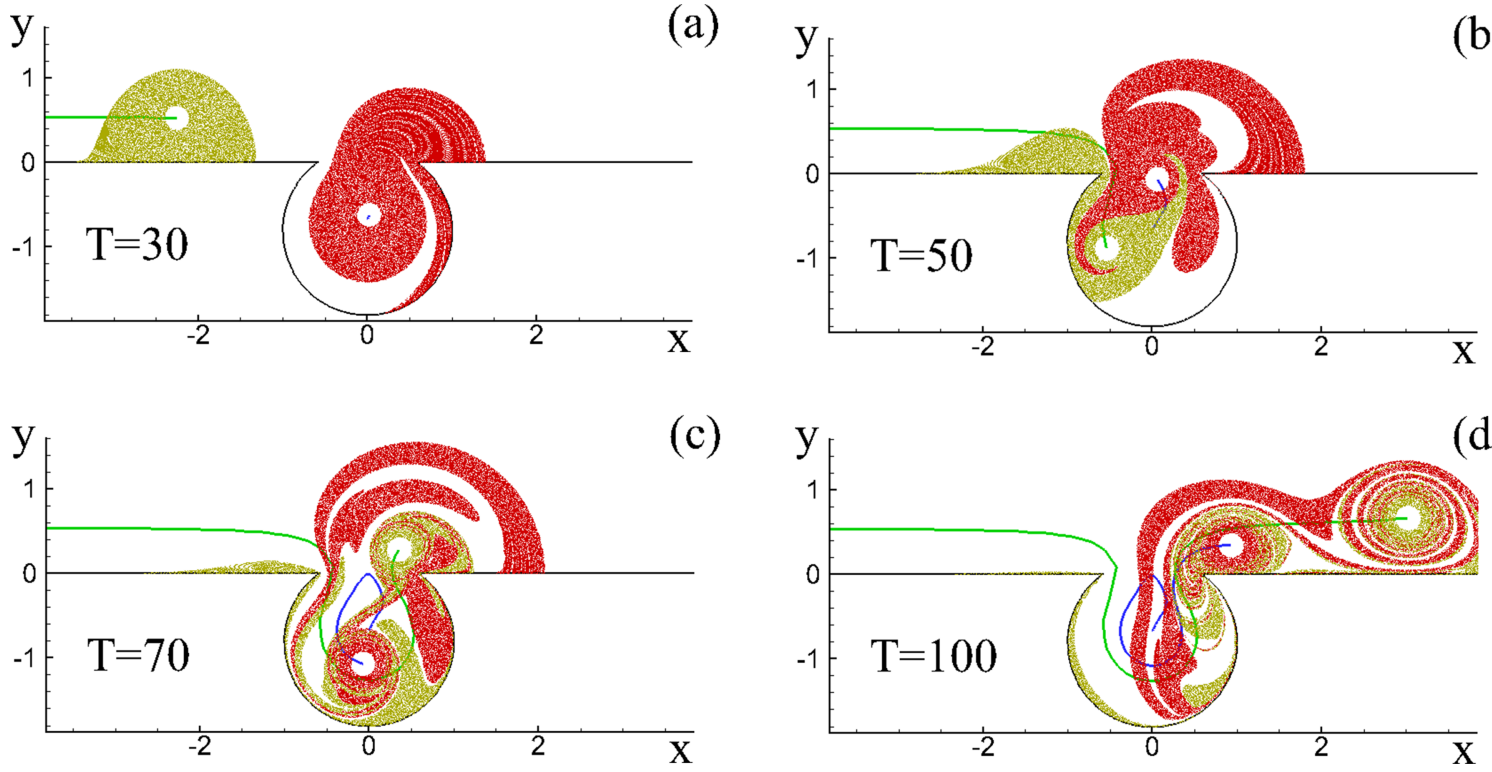

(c)

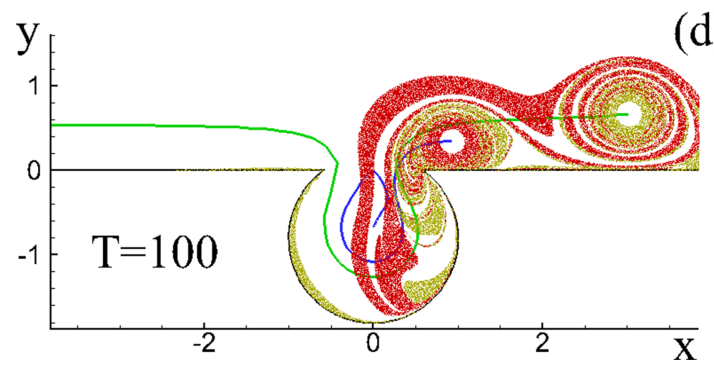

(d)

FIG. 10. The same as in Fig. 9 but for the vortex trajectories shown in Fig. 6(b).

Also $15 \times 10^{4}$ markers are uniformly distributed within the steady-state separatrix of the incident vortex. It is worth recalling that a point vortex moving steadily along a straight boundary induces a closed recirculation zone, where fluid particles move regularly. This recirculation zone is enclosed by the separatrix, which, in the steady state, is a barrier to fluid particle trajectories. Once perturbed, the separatrix vanishes, and fluid particle exchange between the recirculation zone and the exterior current occurs. In the problem under study, the separatrix gets perturbed when the incident vortex comes close to the cavity.

In order to calculate the trajectory of a passive fluid particle, one needs to integrate the following equation:

$$
\begin{aligned}
\frac{d z^{*}}{d t}= & \frac{d \zeta}{d z}\left(\frac{i \mu_{1}}{\zeta(z)-\zeta^{*}\left(z_{1}\right)}-\frac{i \mu_{1}}{\zeta(z)-\zeta\left(z_{1}\right)}+\frac{i \mu_{2}}{\zeta(z)-\zeta^{*}\left(z_{2}\right)}\right. \\
& \left.-\frac{i \mu_{2}}{\zeta(z)-\zeta\left(z_{2}\right)}\right)
\end{aligned}
$$

where $z=x+i y$ is the position of the fluid particle and $z_{1}$, $z_{2}$ are the positions of the cavity-bound and incident vortices calculated using Eq. (2), respectively.

Figures 9-11 illustrate successive instantaneous distributions of the markers for the given running time. Figure 9 corresponds to the initial configuration of the system with parameter $\alpha=0.2304$ and the following initial positions of the vortices: the cavity-bound vortex- $x_{1}(0)=0, y_{1}(0)=-0.41$,
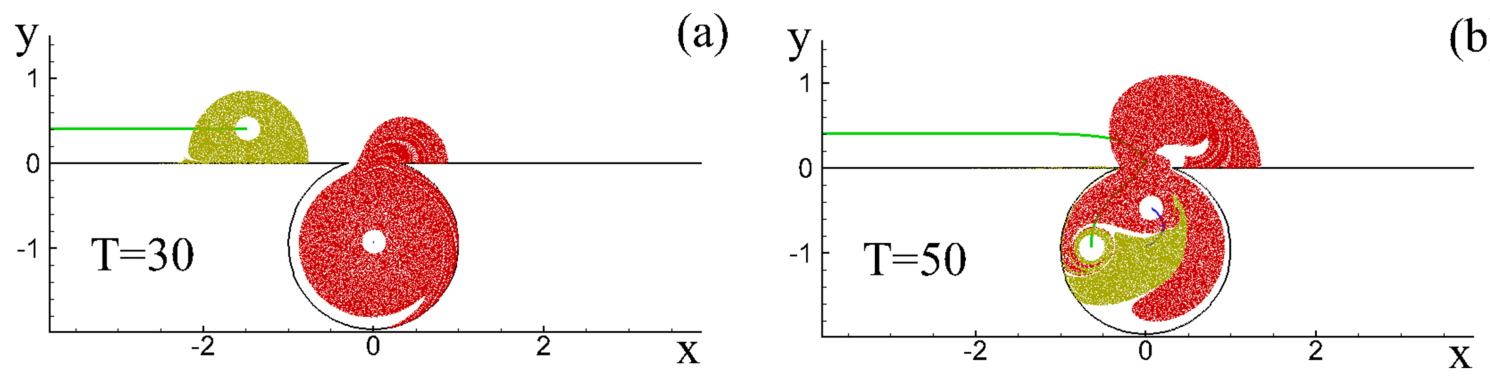

(b)
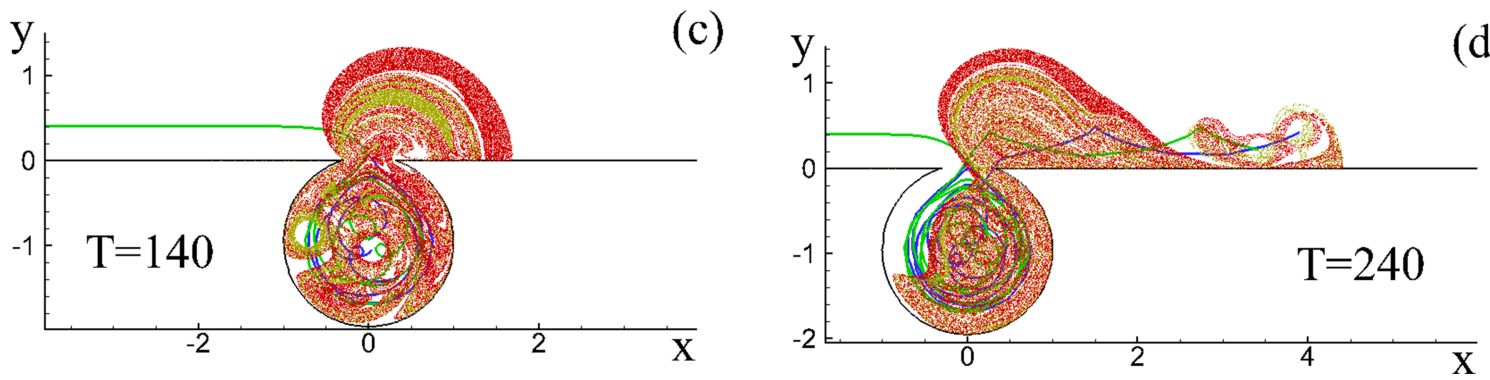

(d)

FIG. 11. The same as in Fig. 9 but for the vortex trajectories shown in Fig. 7(d). 
the incident vortex- $x_{2}(0)=-5, y_{2}(0)=0.7$ as in Fig. 4(c). Figure 10 corresponds to $\alpha=0.2, x_{1}(0)=0, y_{1}(0)=-0.6595$, $x_{2}(0)=-5, y_{2}(0)=0.54$ as in Fig. 6(b). Figure 11 corresponds to $\alpha=0.1, x_{1}(0)=0, y_{1}(0)=-0.925, x_{2}(0)=-5, y_{2}(0)=0.41416$ as in Fig. 7(d). Figure 9 illustrates the process of offsetting the cavity-bound vortex into the unbounded motion. In this case, the interaction between the vortices is weak, so that the exchange between their fluid particles also weak. However, the presence of the cavity forces the incident vortex to lose a significant portion of the markers when the incident vortex moves in the immediate vicinity of the cavity.

Figures 10 and 11 show the cases of moderate and profound mixing efficiency, respectively. In the first case, the vortices interact within the cavity for a short time, partially exchanging their markers, so that, when the vortices leave the cavity, they translate partially mixed markers along the boundary. In the second case, the vortices interact within the cavity for a long time, and therefore their markers are almost uniformly mixed. When the vortices leave the cavity, they form a leap-frogging pair.

\section{CONCLUSIONS}

A simple point-vortex model of two vortices interacting along a straight boundary featuring a circular cavity is introduced as an attempt to take into account the influence of curved boundaries of natural gulfs on the eddies' trajectories in the ocean. Despite being highly simplified, the model still hints at the complexity of vortex dynamics near boundaries even if the vortices are able to maintain their stability and coherence for a prolonged time. In particular, the model indicates that the along-shore propagating vortices can significantly affect the dynamics of bay-entrapped vortices even when the incident vortices pass at a significant distance from the bay. Moreover, the model shows that such vortex interactions generally produce irregular vortex trajectories similar to case of two point vortices embedded in a time-dependent shear flow. ${ }^{58,59}$

An initial state with one of the vortices being initialized within the bay and the second one moving along the straight boundary toward the bay has been analyzed. Escape time distributions reveal complex behavior of the vortices that may result in different outcomes. (i) The incident vortex may pass near the bay not perturbing significantly the cavity-bound vortex, as a result the cavity-bound vortex remains entrapped within the bay. When the incident vortex moves close enough to the boundary, it may pass the bay moving closely to the boundary as if the straight boundary continued. In this case, the influence of the incident vortex on the cavity-bound vortex may also be negligible. (ii) The incident vortex may become entrapped inside the cavity instead of the cavity-bound one, whereas this one leaves the cavity and moves away from it. (iii) Both vortices leave the bay almost not interacting or shortly after the interaction as separate vortices or as a bound leapfrogging pair. (iv) Both vortices may be entrapped within the cavity for a significant amount of time producing complex trajectories.

Possible directions of further study might be as follows. First, in order to increase applicability, the model should take into account the Kutta condition at the sharp cusps. In order to do this, one should introduce additional point vortices near the cusps to create flow separation at the cusps. This may significantly alter the evolution of the system resulting in even more complex dynamics.

Second, the dynamics of the vortices is of considerable interest also if one examines ensuing exchange of fluid particles that accompany the interaction. Indeed, initially both vortices induce regular advection of their surrounding fluid particles. The vortices induce closed recirculation regions in their vicinity; the fluid within these regions does not mix with the ambient fluid (let us recall that there is no molecular diffusion in the model). However, when the vortices start interacting, the dynamics of the fluid particles near the interaction becomes irregular, facilitating stirring with the ambient fluid. Therefore, when vortices leave the cavity, the content of their neighboring fluid is very different from the initial one. A qualitative analysis of the fluid particle mixing ensuing from the interaction might deserve a separate study.

\section{ACKNOWLEDGMENTS}

The reported study was partially supported by the Russian Foundation for Basic Research, Project No. 16-55150001/1069 (jointly with Centre National de la Recherche Scientifique), No. 17-05-00035. E.A.R. was partially supported by the Ministry of Education and Science of Russian Federation, Project No. $M K-172.2017 .1$. The work of K.V.K. in obtaining the analytical estimates was supported by the Russian Scientific Foundation, Project No. 16-1710025. M.A.S. was partially supported by the Ministry of Education and Science of Russian Federation, Project No. N14.W.03.31.0006.

${ }^{1}$ X. Carton, L. Chérubin, J. Paillet, Y. Morel, A. Serpette, and B. L. Cann, "Meddy coupling with a deep cyclone in the Gulf of Cadiz," J. Mar. Syst. 32, 13-42 (2002).

${ }^{2}$ X. Carton, B. Le Cann, A. Serpette, and J. Dubert, "Interactions of surface and deep anticyclonic eddies in the Bay of Biscay," J. Mar. Syst. 109, S45-S59 (2013).

${ }^{3}$ P. L'Hégaret, X. Carton, I. Ambar, C. Menesguen, B. Hua, L. Chérubin, A. Aguiar, B. Le Cann, N. Daniault, and N. Serra, "Evidence of Mediterranean water dipole collision in the Gulf of Cadiz," J. Geophys. Res.: Oceans 119, 5337-5359, https://doi.org/10.1002/2014jc009972 (2014).

${ }^{4}$ B. A. Klinger, "Baroclinic eddy generation at a sharp corner in a rotating system," J. Geophys. Res. 99, 12515-12531, https://doi.org/ 10.1029/93jc03585 (1994).

${ }^{5}$ O. R. Southwick, E. R. Johnson, and N. R. McDonald, "A simple model for sheddies: Ocean eddies formed from shed vorticity," J. Phys. Oceanogr. 46, 2961-2979 (2016).

${ }^{6}$ X. Carton, "Hydrodynamical modeling of oceanic vortices," Surv. Geophys. 22, 179-263 (2001).

${ }^{7}$ V. M. Gryanik, M. A. Sokolovskiy, and J. Verron, "Dynamics of heton-like vortices," Regular Chaotic Dyn. 11, 383-434 (2006).

${ }^{8}$ K. V. Koshel and S. V. Prants, "Chaotic advection in the ocean," Phys.-Usp. 49, 1151-1178 (2006).

${ }^{9}$ O. R. Southwick, E. R. Johnson, and N. R. McDonald, "A point vortex model for the formation of ocean eddies by flow separation," Phys. Fluids 27, 016604 (2015).

${ }^{10}$ M. A. Sokolovskiy and J. Verron, "Finite-core hetons: Stability and interactions," J. Fluid Mech. 423, 127-154 (2000).

${ }^{11}$ E. R. Johnson and N. R. McDonald, "The motion of a vortex near a gap in a wall," Phys. Fluids 16, 462-469 (2004).

${ }^{12}$ E. R. Johnson and N. R. McDonald, "The point island approximation in vortex dynamics," Geophys. Astrophys. Fluid Dyn. 99, 49-60 (2005).

${ }^{13}$ E. R. Johnson and N. R. McDonald, "Vortices near barriers with multiple gaps," J. Fluid Mech. 531, 335-358 (2005). 
${ }^{14}$ M. A. Sokolovskiy and J. Verron, Dynamics of Vortex Structures in a Stratified Rotating Fluid (Springer, Switzerland, 2014).

${ }^{15}$ E. A. Ryzhov and M. A. Sokolovskiy, "Interaction of a two-layer vortex pair with a submerged cylindrical obstacle in a two layer rotating fluid," Phys. Fluids 28, 056602 (2016).

${ }^{16}$ B. Legras and V. Zeitlin, "Conformal dynamics for vortex motions," Phys. Lett. A 167, 265-271 (1992).

${ }^{17}$ D. Crowdy and A. Surana, "Contour dynamics in complex domains," J. Fluid Mech. 593, 235-254 (2007).

${ }^{18}$ V. Makarov and S. Bulgakov, "Regimes of near-wall vortex dynamics in potential flow through gaps," Phys. Fluids 20, 086605 (2008).

${ }^{19} \mathrm{M}$. Duran-Matute and O. U. Velasco-Fuentes, "Passage of a barotropic vortex through a gap,” J. Phys. Oceanogr. 38, 2817-2831 (2008).

${ }^{20}$ N. R. McDonald and E. R. Johnson, "Gap-leaping vortical currents," J. Phys. Oceanogr. 39, 2665-2674 (2009).

${ }^{21}$ I. Gned, H. Telib, and L. Zannetti, "Conformal contour dynamics in bounded domains," Eur. J. Mech. B/Fluids 29, 369-376 (2010).

${ }^{22}$ A. Crosby, E. R. Johnson, and P. J. Morrison, "Deformation of vortex patches by boundaries," Phys. Fluids 25, 023602 (2013).

${ }^{23}$ A. K. Hinds, E. R. Johnson, and N. R. McDonald, "Beach vortices near circular topography," Phys. Fluids 28, 106602 (2016).

${ }^{24} \mathrm{C}$. Lin, "On the motion of vortices in two dimensions. I. Existence of the Kirchhoff-Routh function," Proc. Natl. Acad. Sci. U. S. A. 27, 570-575 (1941).

${ }^{25}$ P. G. Saffman, Vortex Dynamics (Cambridge University Press, Cambridge, 1992).

${ }^{26}$ L. Milne-Thomson, Theoretical Hydrodynamics (Macmillan, London, 1968).

${ }^{27}$ E. A. Ryzhov and K. V. Koshel, "Steady and perturbed motion of a point vortex along a boundary with a circular cavity," Phys. Lett. A 380, 896-902 (2016).

${ }^{28}$ B. V. Chirikov, "A universal instability of many-dimensional oscillator systems," Phys. Rep. 52, 263-379 (1979).

${ }^{29}$ A. J. Lichtenberg and M. A. Lieberman, Regular and Chaotic Dynamics, 2nd ed. (Springer-Verlag, 1992).

${ }^{30}$ G. M. Zaslavsky, Physics of Chaos in Hamiltonian Dynamics (Imperial College Press, London, 1998).

${ }^{31}$ Y. K. Suh, "Periodic motion of a point vortex in a corner subject to a potential flow,” J. Phys. Soc. Jpn. 62, 3441-3445 (1993).

${ }^{32}$ V. F. Kozlov and K. V. Koshel, "Barotropic model of chaotic advection in background flows," Izv., Atmos. Oceanic Phys. 35, 638-648 (1999).

${ }^{33}$ V. F. Kozlov and K. V. Koshel, "A model of chaotic transport in the barotropic background flow," Izv., Atmos. Oceanic Phys. 36, 119-128 (2000).

${ }^{34}$ B. R. Noack, I. Mezic, G. Tadmor, and A. Banaszuk, "Optimal mixing in recirculation zones," Phys. Fluids 16, 867-888 (2004).

${ }^{35}$ W. K. Lee, P. H. Taylor, A. G. L. Borthwick, and S. Chuenkhum, "Vortexinduced chaotic mixing in wavy channels," J. Fluid Mech. 654, 501-538 (2010).

${ }^{36}$ K. Shariff and A. Leonard, "Vortex rings," Annu. Rev. Fluid Mech. 24, 235-279 (1992).

${ }^{37}$ K. Shariff, A. Leonard, and J. H. Ferziger, "Dynamical systems analysis of fluid transport in time-periodic vortex ring flows," Phys. Fluids 18, 047104 (2006).

${ }^{38}$ A. Péntek, T. Tél, and Z. Toroczkai, "Chaotic advection in the velocity-field of leapfrogging vortex pairs,” J. Phys. A: Math. Gen. 28, 21911-22216 (1995).
${ }^{39}$ L. Tophoj and H. Aref, "Instability of vortex pair leapfrogging," Phys. Fluids 25, 014107 (2013).

${ }^{40}$ H. Aref, "Stirring by chaotic advection," J. Fluid Mech. 143, 1-21 (1984).

${ }^{41}$ V. Rom-Kedar, A. Leonard, and A. Wiggins, "An analytical study of transport, mixing and chaos in an unsteady vortical flow," J. Fluid Mech. 214, 347-394 (1990).

${ }^{42}$ L. Kuznetsov and G. M. Zaslavsky, "Regular and chaotic advection in the flow field of a three-vortex system," Phys. Rev. E 58, 7330-7349 (1998).

${ }^{43}$ L. Kuznetsov and G. M. Zaslavsky, "Passive particle transport in threevortex flow," Phys. Rev. E 61, 3777-3792 (2000).

${ }^{44} \mathrm{H}$. Aref, "The development of chaotic advection," Phys. Fluids 14, 13151325 (2002)

${ }^{45}$ M. Budyansky, M. Uleysky, and S. Prants, "Hamiltonian fractals and chaotic scattering of passive particles by a topographical vortex and an alternating current," Phys. D 195, 369-378 (2004).

${ }^{46}$ V. F. Kozlov, K. V. Koshel, and D. V. Stepanov, "Influence of the boundary on chaotic advection in the simplest model of a topographic vortex," Izv., Atmos. Ocean. Phys. 41, 217-227 (2005).

${ }^{47}$ Y. G. Izrailsky, K. V. Koshel, and D. V. Stepanov, "Determination of optimal excitation frequency range in background flows," Chaos 18, 013107 (2008).

${ }^{48}$ E. Ryzhov, K. Koshel, and D. Stepanov, "Background current concept and chaotic advection in an oceanic vortex flow," Theor. Comput. Fluid Dyn. 24, 59-64 (2010).

${ }^{49}$ E. A. Ryzhov, "On changing the size of the atmosphere of a vortex pair embedded in a periodic external shear flow," Phys. Lett. A 375, 3884-3889 (2011).

${ }^{50}$ E. A. Ryzhov, K. V. Koshel, and X. J. Carton, "Passive scalar advection in the vicinity of two point vortices in a deformation flow," Eur. J. Mech. B/Fluids 34, 121-130 (2012).

${ }^{51}$ G. Drotos, T. Tel, and G. Kovacs, "Modulated point-vortex pairs on a rotating sphere: Dynamics and chaotic advection," Phys. Rev. E 87, 063017 (2013).

${ }^{52}$ K. V. Koshel, M. A. Sokolovskiy, and J. Verron, "Three-vortex quasigeostrophic dynamics in a two-layer fluid. Part 2. Regular and chaotic advection around the perturbed steady states," J. Fluid Mech. 717, 255-280 (2013).

${ }^{53}$ R. M. Samelson, "Lagrangian motion, coherent structures, and lines of persistent material strain,” Annu. Rev. Mar. Sci. 5, 137-163 (2013).

${ }^{54}$ E. A. Ryzhov, "Irregular mixing due to a vortex pair interacting with a fixed vortex,” Phys. Lett. A 378, 3301-3307 (2014).

${ }^{55} \mathrm{G}$. Drotos and T. Tel, "On the validity of the beta-plane approximation in the dynamics and the chaotic advection of a point vortex pair model on a rotating sphere," J. Atmos. Sci. 72, 415-429 (2015).

${ }^{56}$ E. A. Ryzhov and K. V. Koshel, "Resonance phenomena in a two-layer two-vortex shear flow," Chaos 26, 113116 (2016).

${ }^{57}$ H. Aref, J. R. Blake, M. Budisic, S. S. S. Cardoso, J. H. E. Cartwright, H. J. H. Clercx, K. El Omari, U. Feudel, R. Golestanian, E. Gouillart, G. F. van Heijst, T. S. Krasnopolskaya, Y. Le Guer, R. S. MacKay, V. V. Meleshko, G. Metcalfe, I. Mezic, A. P. S. de Moura, O. Piro, M. F. M. Speetjens, R. Sturman, J.-L. Thiffeault, and I. Tuval, "Frontiers of chaotic advection," Rev. Mod. Phys. 89, 025007 (2017).

${ }^{58} \mathrm{X}$. Perrot and X. Carton, "Point-vortex interaction in an oscillatory deformation field: Hamiltonian dynamics, harmonic resonance and transition to chaos," Discrete Contin. Dyn. Syst.-Ser. B 11, 971-995 (2009).

${ }^{59}$ E. A. Ryzhov and K. V. Koshel, "Two-point-vortex evolution in an oscillatory shear flow with rotation,” EPL (Europhys. Lett.) 108, 24002 (2014). 